\title{
Auf kognitive Störungen achten
}

_ Die Hepatische Enzephalopathie (HE) ist eine zerebrale Funktionsstörung mit schubförmigem Verlauf, die sich auf dem Boden einer Leberzirrhose entwickeln kann. Anhaltende Müdigkeit, Konzentrationsstörungen, Gedächtnisschwäche und psychomotorische Auffälligkeiten sind die ersten Anzeichen, berichtete Dr. Marc Nguyen-Tat, Mainz. Ohne Therapie verschlimmern sich die Symptome und können über Desorientiertheit zu Leberkoma und Tod führen. Wegen der unspezifischen Beschwerden bleibt eine subklinische HE häufig unerkannt, obschon die Lebensqualität bereits deutlich eingeschränkt ist.

Die Pathogenese der HE beruht auf einem Zusammenspiel verschiedener Faktoren. Eine Schlüsselrolle kommt der eingeschränkten Entgiftungsleistung der Leber zu. Dadurch gelangen toxische Stoffwechselprodukte, z. B. Ammoniak, in das Gehirn. Die Erkran- kung ist potenziell reversibel, sofern es gelingt, die Anreicherung dieser Substanzen im Gehirn zu unterbinden.

\section{Konsequente Rezidivprophylaxe}

Patienten mit Leberzirrhose müssen gezielt auf Symptome einer HE untersucht und sorgfältig überwacht werden. Bei nur einer Episode einer höhergradigen HE liegt das Risiko der Patienten, innerhalb der folgenden 12 Monate zu versterben, bei über 50\% [Cordoba J et al. J Hepatol. 2014;60:275-81]. Mit jeder weiteren Episode können die kognitiven Defizite des Patienten zunehmen [Bajaj JS et al. Gastroenterol. 2010;138:2332-40]. Da die Erkrankung potenziell reversibel ist, sei es umso wichtiger, bei nach der ersten HEEpisode eine konsequente Rezidivprophylaxe durchzuführen, so Nguyen-Tat.

Standard in der HE-Rezidivprophylaxe ist die Behandlung mit Rifaximin- $\alpha$ (Xifaxan $550 \mathrm{mg}$ ). Das schwer resorbier-

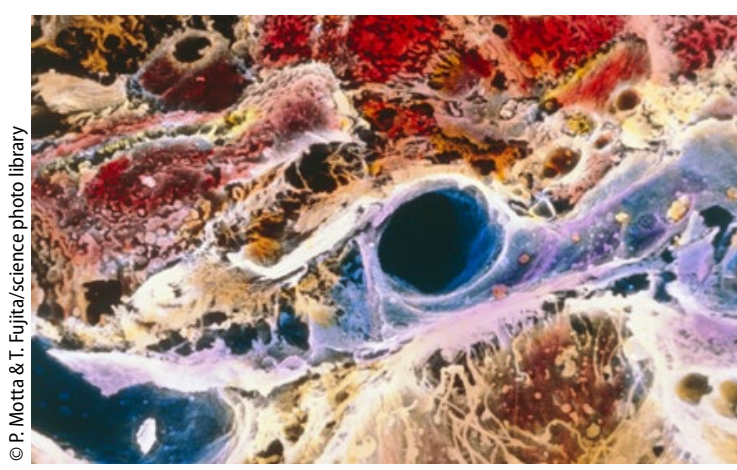

Leberzirrhose: kolorierte elektronenmikroskopische Aufnahme.

bare Antibiotikum wirkt fast ausschließlich im Darm, wo es durch seine bakterizide Wirkung auf ammoniakproduzierende Bakterien kausal in die Pathogenese der HE eingreift. Unter konsequenter Langzeittherapie verringert Rifaximin- $\alpha$ $(2 \times 550 \mathrm{mg} / \mathrm{d})$ die HE-Rezidivrate über sechs Monate um mehr als die Hälfte im Vergleich zu Placebo.

Abdol A. Ameri

- Fachpressegespräch „Lebererkrankungen erkennen, therapieren und begleiten", DGIM 2017; Mannheim, Mai 2017 (Veranstalter: Norgine)

\section{Akute Bronchitis}

\section{Prolongiertem Husten vorbeugen}

— Husten und Hustenreiz verstärken bei fast der Hälfte der Betroffenen die Wahrnehmung der anderen Symptome einer akuten Atemwegsinfektion. Zudem berichten viele Patienten, dass das Husten oft auch noch anhielt, wenn die anderen Erkältungssymptome bereits abgeklungen waren.

Der Hustenreiz bei einer Atemwegsinfektion entsteht nach derzeitigem Wissensstand durch die Aktivierung von Hustenrezeptoren im Bereich des Kehlkopfes und der zentralen Bronchien [Kardos P et al. Pneumologie. 2010; 64:336-73]. Dabei gilt es inzwischen als gesichert, dass die Entzündung der Bronchialschleimhaut und eine damit verbundene Aktivierung und Sensibili- sierung entsprechender Rezeptoren hierbei eine entscheidende Rolle spielen.

Die moderne Behandlung bei akuter Bronchitis besteht daher nicht nur aus dem Einsatz sekretolytisch wirkender Substanzen, sondern sollte zudem eine antiphlogistische Komponente beinhalten. Beides bietet die pflanzliche Extrakt-Kombination Bronchipret ${ }^{\circledR}$ : Der Thymianextrakt wirkt antiphlogistisch, während Auszüge der enthaltenen Saponindrogen Efeu und Primel als Expektoranzien dienen. Die entzündungshemmenden Eigenschaften wurden in vivo belegt [Seibel J et al. ERJ. 2014;44(Suppl 58):P1794].

Red.

- Nach Informationen von Bionorica

\section{Vereinfachte Schmerztherapie}

Starke Schmerzen, wie sie z.B. nach einem Bandscheibenvorfall, bei Arthrose, Radikulopathien oder Spinalkanalstenosen auftreten, sind neben neuropathischen Schmerzen oder Tumorschmerzen häufige Indikationen für den Einsatz der retardierten Fixkombination aus Oxycodon/Naloxon. Seit der Erhöhung der Tagesdosis von Targin ${ }^{\circledast}$ (160 mg/80 $\mathrm{mg}$ ) ist ein entsprechender Spielraum für die Auftitration gemäß der Schmerzstärke geschaffen worden. Seit 18. April 2017 liegt die Fixkombination aus retardiertem Oxycodon und retardiertem Naloxon in den höheren Wirkstärken $60 \mathrm{mg} / 30 \mathrm{mg}$ und $80 \mathrm{mg} / 40 \mathrm{mg}$ vor. Diese Wirkstärken sind an die Tageshöchstdosis angepasst und erleichtern die Therapie stärkster Schmerzen durch eine einfachere Handhabung, weil nun die Gabe von $2 \times 60 \mathrm{mg} / 30 \mathrm{mg} \mathrm{bzw} .2 \times 80 \mathrm{mg} / 40 \mathrm{mg}$ möglich ist.

Red. 